\title{
Neonatal anthropometry: a tool to evaluate the nutritional status and predict early and late risks
}

- $\quad$ Luis Pereira-da-Silva

Purchase on Springer.com (\$29.95 / €24.95 / £19.95)

\section{Abstract}

Neonatal anthropometry is an inexpensive, noninvasive and convenient tool for bedside evaluation, especially in sick and fragile neonates. Anthropometry can be used in neonates as a tool for several purposes: diagnosis of foetal malnutrition and prediction of early postnatal complications; postnatal assessment of growth, body composition and nutritional status; prediction of long-term complications including metabolic syndrome; assessment of dysmorphology; and estimation of body surface. However, in this age group anthropometry has been notorious for its inaccuracy and the main concern is to make validated indices available. Direct measurements, such as body weight, length and body circumferences are the most commonly used measurements for nutritional assessment in clinical practice and in field studies. Body weight is the most reliable anthropometric measurement and therefore is often used alone in the assessment of the nutritional status, despite not reflecting body composition. Derived indices from direct measurements have been proposed to improve the accuracy of anthropometry. Equations based on body weight and length, mid-arm circumference/head circumference ratio, and upper-arm cross-sectional areas are among the most used derived indices to assess nutritional status and body proportionality, even though these indices require further validation for the estimation of body composition in neonates.
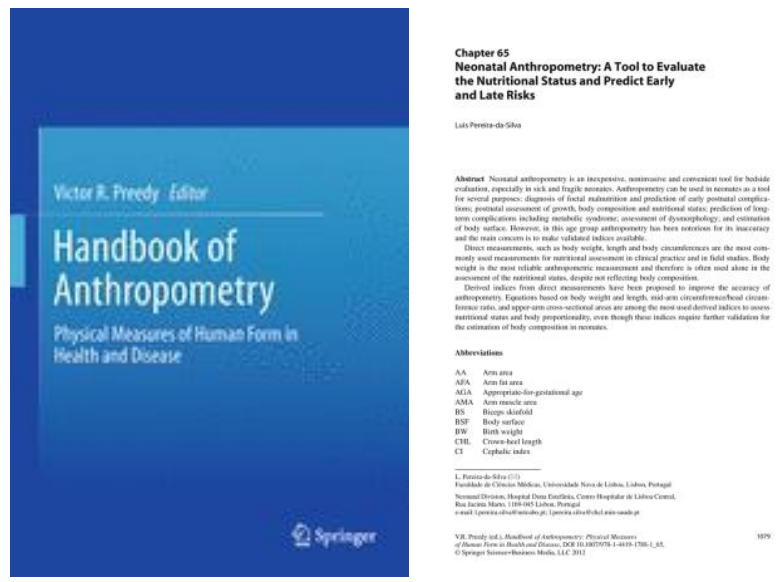


\section{References (45)}

1. Ananth CV, Vintzileos AM, Shen-Schwarz S, Smulian JC, Lai YL Obstet Gynecol. 1998;91:917-24. CrossRef

2. Beattie RB, Johnson P Br J Obstet Gynaecol. 1994;101:842-6. CrossRef

3. Current JD. Internet J Anesthesiol. 1998;2(2).

4. Dangerfield PH, Taylor CJ Early Hum Dev. 1983;8:225-33. CrossRef

5. De Bruin NC, Van Velthoven KAM, Stijnen T, Juttmann RE, Degenhart HJ, Visser HKA Am J Clin Nutr. 1995;61:1195-205.

6. Ehrenkranz RA, Younes N, Lemons JA, Fanaroff AA, Donovan EF, Wright LL, Katsikiotis V, Tyson JE, Oh W, Shankaran S, Bauer CR, Korones SB, Stoll BJ, Stevenson DK, Papile LA Pediatrics. 1999;104:280-9. CrossRef

7. Engstrom E, Wallgren K, Hellstrom A, Niklasson A Acta Paediatr. 2003;92:211-5. CrossRef

8. Fenton TR. BMC Pediatr. 2003;3:13. CrossRef

9. Fletcher MA. In: Fletcher MA, editor. Physical diagnosis in neonatology. Philadelphia, PA: Lippincott-Raven; 1998; pp. 29-54.

10. Gibson AT, Carney S, Wright NP, Wales JKN Horm Res. 2003;59 (suppl 1):119-28. CrossRef

11. Jaquet $D$, Deghmoun $S$, Chevenne D, Collin D, Czernichow P, Levy-Marchal C Diabetologia. 2005;48:849-55. CrossRef

12. Jellifee EFP, Jelliffee DB. The arm circumference as a public health index of proteincalorie malnutrition of early childhood. J Trop Pediatr. 1969;32:1527-30. CrossRef

13. Johnson TS, Engstrom JL, Gelhar DK J Pediatr Gastroenterol Nutr. 1997;24:497-505. CrossRef

14. Koo WW, Walters JC, Hockman EM J Nutr. 2000;130:2188-94.

15. Koo WW, Walters JC, Hockman EM Pediatr Res. 2004;56:694-700. CrossRef

16. Lubchenco LO, Hansman C, Boyd E Pediatrics. 1966;37:403-8.

17. Meban C. J Anat. 1983;137:271-8.

18. Olhager E, Forsum E Acta Paediatr. 2006;95:21-8. CrossRef

19. Olsen IE, Richardson DK, Schmid CH, Ausman LM, Dwyer JT Pediatrics. 2002;110:112532. CrossRef

20. Olsen IE, Lawson ML, Mainzen-Derr J, Sapsford AL J Pediatr. 2009;154:486-91. CrossRef

21. Olsen IE, Groveman SA, Lawson ML, Clark RH, Zemel BS Pediatrics. 2010;125;e214e24. CrossRef

22. Owen P. Clin Exp Obstet Gynecol. 1997;24:8-10.

23. Patterson RM, Pouliot MR Am J Obstet Gynecol. 1987;157:691-3.

24. Patterson RM, Pouliot MR. Neonatal morphometrics and perinatal outcome: who is growth retarded? Am J Obstet Gynecol 1987;157:691-3.

25. Paulsson L, Bondemark L Angle Orthod. 2009;79:276-83. CrossRef

26. Pereira-da-Silva L, Gomes JV, Clington A, Videira Amaral JM, Bustamante SA Early Hum Dev. 1999;54:117-28. CrossRef

27. Pereira-da-Silva L, Bergmans KIM, van Kerkhoven LAS, Leal F, Virella D, Videira-Amaral JM Acta Paediatr. 2006;95:742-6. CrossRef

28. Pereira-da-Silva L, Virella D, Amaral JMV, Guerra A Anthropometry in the newborn: revisited and updated. Lisbon: Nestlé Nutrition Institute(edited in Portuguese); 2007.

29. Pereira-da-Silva L, Abecasis F, Virella D, Videira-Amaral JM Neonatology. 2009;95:74-9. CrossRef

30. Prins I. Pediatric Rev Commun. 1995;8:157-70. 
31. Rodríguez G, Samper MP, Ventura P, Moreno LA, Olivares JL, Pérez-González JM Eur J Pediatr. 2004;163:457-61. CrossRef

32. Rolland-Cachera MF, Brambilla P, Manzoni P, Akrout M, Sironi S, Del Maschio A, Chiumello G Am J Clin Nutr. 1997;65:1709-13.

33. Rubin LP. Postnatal growth in preterm infants: too small, too big, or just right? J Pediatr 2009;154:473-5.

34. Sann L, Durand M, Picard J, Lasne Y, Bethenod M Arch Dis Child. 1988;63:256-60. CrossRef

35. Sasanow SR, Georgieff MK, Pereira G J Pediatr. 1986;109:311-5. CrossRef

36. Schmelzle HR, Fusch C. Am J Clin Nutr. 2002;76:1096-100.

37. Shinwell ES, Shlomo M. Measured length of normal term infants changes over the first two days of life. J Pediatr Endocrinol Metab. 2003;16:537-40. CrossRef

38. Shinwell ES, Shlomo M J Pediatr Endocrinol Metab. 2003;16:537-40. CrossRef

39. Tamim H, Beydoun H, Itani M, Khogali M, Chokr I, Yunis KA; National Collaborative Perinatal Neonatal Network. J Perinat Med. 2004;32:509-13. CrossRef

40. Thornton CJ, Shannon DL, Hunter MA, Brans YW Pediatr Res. 1982;16:989-94. CrossRef

41. Uhing MR, Das UG Clin Perinatol. 2009;36:165-76. CrossRef

42. Uthaya S, Thomas EL, Hamilton G, Dore CJ, Bell J, Modi N Pediatr Res. 2005;57:211-5. CrossRef

43. Wilcox MA, Johnson IR, Maynard PV, Smith SJ, Chilvers CE Br J Obstet Gynaecol. 1993;100:342-7. CrossRef

44. Yau KI, Chang MH Acta Paediatr. 1993;82:427-9. CrossRef

45. Zankl A. Pediatrics. 2004;114:e333-6. CrossRef

\section{About this Chapter}

Title: Neonatal Anthropometry: A Tool to Evaluate the Nutritional Status and Predict Early and Late Risks

Book Title: $\underline{\text { Handbook of Anthropometry }}$

Book Subtitle: Physical Measures of Human Form in Health and Disease

Pages: pp 1079-1104

Copyright: 2012

DOI: 10.1007/978-1-4419-1788-1_65

Print ISBN: 978-1-4419-1787-4

Online ISBN: 978-1-4419-1788-1

Publisher: Springer New York

Copyright Holder: Springer Science+Business Media, LLC

Topics: Health Informatics, Maternal and Child Health, Health Promotion and Disease Prevention, Clinical Nutrition

Editors: Victor R. Preedy $\square^{(\mathrm{ID} 1)}$

Editor Affiliations: ID1. Dept. Nutrition \& Dietetics, King's College

Authors: Luis Pereira-da-Silva $\square^{(1)(2)}$

Author Affiliations: 1. Faculdade de Ciências Médicas, Universidade Nova de Lisboa, Lisbon, Portugal; 2. Neonatal Division, Hospital Dona Estefânia, Centro Hospitalar de Lisboa Central, Rua Jacinta Marto, 1169-045, Lisbon, Portugal 